\title{
On the $S$-matrix of Liouville theory
}

\author{
George Jorjadze ${ }^{a, b, c}$ and Stefan Theisen ${ }^{c}$ \\ ${ }^{a}$ Free University of Tbilisi, \\ Agmashenebeli Alley 240, 0159, Tbilisi, Georgia \\ ${ }^{b}$ Razmadze Mathematical Institute of TSU, \\ Tamarashvili 6, 0177, Tbilisi, Georgia \\ ${ }^{c}$ Max-Planck-Institut für Gravitationsphysik, Albert-Einstein-Institut, \\ 14476, Golm, Germany \\ E-mail: g.jorjadze@freeuni.edu.ge, theisen@aei.mpg.de
}

ABSTRACT: The $S$-matrix for each chiral sector of Liouville theory on a cylinder is computed from the loop expansion of correlation functions of a one-dimensional field theory on a circle with a non-local kinetic energy and an exponential potential. This action is the Legendre transform of the generating function of semiclassical scattering amplitudes. It is derived from the relation between asymptotic in- and out-fields. Its relevance for the quantum scattering process is demonstrated by comparing explicit loop diagrams computed from this action with other methods of computing the $S$-matrix, which are also developed.

KEywords: Conformal Field Theory, Field Theories in Lower Dimensions, Integrable Field Theories

ArXIV EPRINT: 2011.06876 


\section{Contents}

1 Introduction and summary 1

2 Liouville dynamics and asymptotic fields 3

3 Canonical structure $\quad 5$

4 Semiclassical $\boldsymbol{S}$-matrix $\quad 8$

4.1 Semiclassical amplitudes $1 \quad 9$

$\begin{array}{lll}4.2 & \text { Relation to classical one-dimensional field theory } & 10\end{array}$

$\begin{array}{lll}4.3 & \text { Semiclassical amplitudes } 2 & 14\end{array}$

5 Quantum $S$-matrix $\quad 16$

$\begin{array}{ll}5.1 \text { Quantum amplitudes 1 } & 16\end{array}$

$\begin{array}{lll}5.2 & \text { Quantum amplitudes 2 } & 18\end{array}$

6 Conclusions 20

$\begin{array}{ll}\text { A Chiral symplectic forms } & 21\end{array}$

B Generating function as action functional $\quad 22$

\section{Introduction and summary}

Liouville theory is one of the most interesting and most studied interacting two-dimensional conformal field theories. It plays a central role in non-critical bosonic string theory where it arises when one integrates the Weyl anomaly of the Polyakov action in a non-trivial metric background in conformal gauge [1]. There are several reviews which cover the subject very well and we refer to them for further motivations and thorough discussions [2-4]. Various generalisations have been considered, in particular supersymmetric ones, but also to higher dimensions [5]. Here we will only discuss the two-dimensional bosonic theory. We concentrate on a particular aspect of Liouville theory, namely the $S$-matrix of scattering states, a subject which was initiated in [6]. An expression for the $S$-matrix in closed form is not known and the purpose of this note is to report an observation which allows for a reformulation of the problem, which might shed a new light on the issue.

The dynamics of Liouville theory is derived from the Liouville action. The classical equations of motion can be solved and the general solution is specified by two arbitrary functions. They are defined, respectively, on the two branches of the light cone or, in the Euclidean formulation of the theory, by an holomorphic and an anti-holomorphic function. These functions have to respect some regularity conditions and, as we will consider the 
theory on a cylinder, a periodicity requirement as well. The only regular solutions on the cylinder are scattering solutions which interpolate between free asymptotic in- and free asymptotic out-states. The transformation from in- to out-states is effected by the $S$-matrix.

Our aim in this paper is to compute the quantum $S$-matrix of Liouville theory on the cylinder with Minkowski signature. We proceed in two steps. In the first step we compute the semiclassical $S$-matrix or, more precisely, the generating functional $\mathcal{F}$ for semiclassical $S$-matrix elements in the Fock-space generated by the oscillator modes. It is the generating function of the canonical transformation between the $i n$ - and out-fields which are free fields and therefore split into two chiral sectors. We show that this transformation is canonical for each chiral sector separately, with generating functionals $F$ and $\bar{F}$. The two sectors are only coupled through the zero-modes. In terms of the oscillator modes of the $i n$ - and out-fields, in each sector it is a function of the positive modes of the former and of the negative modes of the latter and, of course, of the zero mode, i.e. the momentum. As we will explain in detail, the positive and negative modes in each chiral sector form conjugate pairs which are related by a Legendre transformation with generating function $F$, and, as usually, the momentum is conjugate to the position.

A slight but crucial modification of $F$, which affects only the two-point functions, leads to a re-organization of the conjugate pairs in such a way that we can compute its Legendre transformation. This is essentially just a rewriting of the known relation between the inand out-fields. The Legendre transform, which we call $S$, has the form of a one-dimensional classical action with an unusual non-local kinetic energy term and an exponential potential and where the momentum now plays the role of a coupling constant. We claim that this action, when quantized, contains all the information about the quantum scattering in one chiral sector. A priori this is not clear as the relevance of this action for the quantum theory is not obvious. We support our claim by first computing loop diagrams, where the only regularization required is 'normal ordering' of the potential. We compare these loop results with those obtained from another way to compute scattering amplitudes. This method, which works for the semiclassical and the quantum case, uses the fact that the improved energy-momentum tensors for the $i n$ - and out-fields in each chiral sector coincide. Equating them and expressing the resulting equation in terms of the independent canonical variables, leads to a differential equation for the generating functional which can be solved in a power series in the modes. We use this second method, which for the quantum amplitudes is more efficient than the loop computations and produces results which are valid to all powers in $\hbar$, to check the Feynman diagram computations. We also find agreement with the results obtained by Zamolodchikov \& Zamolodchikov in [6].

The organization of the paper is as follows. In section 2 we review known facts about classical Liouville theory on the cylinder, which will be relevant for the subsequent discussion. Next, in section 3, we discuss the canonical structure and introduce generating functions for canonical transformations between the $i n$ - and out-fields. The most relevant is the transformation between in- and out-oscillator modes, which is canonical in each chiral sector separately. Its generating function $F$ completely specifies the semiclassical $S$-matrix in one chiral sector. Further details about the canonical structure are given in 
two appendices. $F$ will be computed in section 4 , where the relation between the classical in- and out-fields is used to find $S$, the Legendre transform of $F$. We use it to compute semiclassical scattering amplitudes via tree-level Feynman diagrams derived from $S$ and compare them with a direct computation, which exploits the equality between the improved energy-momentum tensors of the asymptotic in- and out-states. In section 5 we compute quantum corrections to the scattering amplitudes. We first do this by evaluating (mostly) one-loop Feynman diagrams and then compare them to an explicit computation which again uses the equality of the in and out energy-momentum tensors, with the quantum corrections to the improvement term taken into account. We end with short conclusions.

\section{Liouville dynamics and asymptotic fields}

We study Liouville theory on a cylinder with spacetime coordinates $\sigma \in \mathbb{S}^{1}$ and $\tau \in \mathbb{R}^{1}$. The classical theory is described by the Liouville equation

$$
\partial_{\tau}^{2} \Phi-\partial_{\sigma}^{2} \Phi+4 \mu^{2} e^{2 \Phi}=0
$$

$\Phi$ is called the Liouville field and $\mu^{2}>0$ is a 'cosmological' constant.

We also introduce the Liouville field exponential $V=e^{-\Phi}$ and the stress tensor components, which in chiral coordinates $(x=\tau+\sigma, \bar{x}=\tau-\sigma)$ are defined as

$$
T=\left(\partial_{x} \Phi\right)^{2}-\partial_{x}^{2} \Phi, \quad \bar{T}=\left(\partial_{\bar{x}} \Phi\right)^{2}-\partial_{\bar{x}}^{2} \Phi .
$$

The chirality conditions $\partial_{\bar{x}} T=0, \partial_{x} \bar{T}=0$ follow from the Liouville equation (2.1). By (2.2) $V$ satisfies the pair of Hill equations

$$
\partial_{x}^{2} V(x, \bar{x})=T(x) V(x, \bar{x}), \quad \partial_{\bar{x}}^{2} V(x, \bar{x})=\bar{T}(\bar{x}) V(x, \bar{x}),
$$

while (2.1), in addition, leads to

$$
V \partial_{x} \partial_{\bar{x}} V-\partial_{x} V \partial_{\bar{x}} V=\mu^{2}
$$

Setting $T=\bar{T}=\frac{1}{4} p^{2}>0$, one finds a simple solution of (2.3) and (2.4)

$$
V=e^{-\Phi}=\frac{\mu}{p}\left(e^{-p \tau}+e^{p \tau}\right),
$$

with $p=\sqrt{p^{2}}$ and $\mu=\sqrt{\mu^{2}}$. The corresponding $\sigma$-independent Liouville field $\Phi$ describes particle dynamics in an exponential potential and $p$ is interpreted as the momentum of the in-coming particle, while the momentum of the out-going particle is $-p$.

The space of solutions of (2.4) is invariant under conformal transformations

$$
V(x, \bar{x}) \mapsto \tilde{V}(x, \bar{x})=\left(\zeta^{\prime}(x) \bar{\zeta}^{\prime}(\bar{x})\right)^{-\frac{1}{2}} V(\zeta(x), \bar{\zeta}(\bar{x})),
$$

where the chiral functions are monotonic $\left(\zeta^{\prime}>0, \bar{\zeta}^{\prime}>0\right)$ and allow the mode expansion

$$
\zeta(x)=\zeta_{0}+x+i \sum_{n \neq 0} \frac{\zeta_{n}}{n} e^{-i n x}, \quad \bar{\zeta}(\bar{x})=\bar{\zeta}_{0}+\bar{x}+i \sum_{n \neq 0} \frac{\bar{\zeta}_{n}}{n} e^{-i n \bar{x}} .
$$


The conformal transformations of (2.5) generate all regular Liouville fields on the cylinder [7]. The general solution can then be written as

$$
e^{-\Phi}=e^{-\Phi_{\text {in }}}+e^{-\Phi_{\text {out }}},
$$

where the fields $\Phi_{\text {in }}$ and $\Phi_{\text {out }}$ are parametrised as follows:

$$
\begin{aligned}
& \Phi_{\text {in }}=\frac{1}{2} \log \left[\xi^{\prime}(x) \bar{\xi}^{\prime}(\bar{x})\right]+\frac{\mu}{2}[\xi(x)+\bar{\xi}(\bar{x})], \\
& \Phi_{\text {out }}=\frac{1}{2} \log \left[\xi^{\prime}(x) \bar{\xi}^{\prime}(\bar{x})\right]-\frac{\mu}{2}[\xi(x)+\bar{\xi}(\bar{x})],
\end{aligned}
$$

with

$$
\xi(x)=\frac{p}{\mu} \zeta(x), \quad \bar{\xi}(\bar{x})=\frac{p}{\mu} \bar{\zeta}(\bar{x}) .
$$

The in and out notation will now be justified.

Due to (2.7), the non-periodic $\tau$-dependent parts of $\Phi_{\text {in }}$ and $\Phi_{\text {out }}$ are $p \tau$ and $-p \tau$, respectively. Since $p>0$, one can neglect $\exp \left(-\Phi_{\text {out }}\right)$ in $(2.8)$ at $\tau \rightarrow-\infty$ and $\exp \left(-\Phi_{\text {in }}\right)$ at $\tau \rightarrow+\infty$. Hence, the fields $\Phi_{\text {in }}(\tau, \sigma)$ and $\Phi_{\text {out }}(\tau, \sigma)$ are interpreted as the asymptotic fields of Liouville theory. They are $2 \mathrm{~d}$ massless free scalar fields, since they are given as the sum of the chiral and antichiral parts

$$
\Phi_{\text {in }}=\phi_{\text {in }}(x)+\bar{\phi}_{\text {in }}(\bar{x}), \quad \Phi_{\text {out }}=\phi_{\text {out }}(x)+\bar{\phi}_{\text {out }}(\bar{x}),
$$

with

$$
\begin{aligned}
\phi_{\text {in }}(x) & =\frac{1}{2} \log \left[\xi^{\prime}(x)\right]+\frac{\mu}{2} \xi(x), \\
\phi_{\text {out }}(x) & =\frac{1}{2} \log \left[\xi^{\prime}(x)\right]-\frac{\mu}{2} \xi(x) .
\end{aligned}
$$

The antichiral part is similar and it suffices to analyze the chiral part only.

The integration of (2.12) defines $\xi(x)$ through the asymptotic fields

$$
e^{\mu \xi(x)}=\mu A(x), \quad e^{-\mu \xi(x)}=\mu B(x),
$$

with

$$
A(x)=\int_{-\infty}^{x} \mathrm{~d} y e^{2 \phi_{\text {in }}(y)}, \quad B(x)=\int_{x}^{\infty} \mathrm{d} y e^{2 \phi_{\text {out }}(y)} .
$$

The integrated free-field exponents $A(x)$ and $B(x)$ are called screening charges. They are conformal scalars similarly to the chiral field $\zeta(x)$. By (2.13) one has the functional relation between the asymptotic fields, $\mu^{2} A(x) B(x)=1$.

Note that the screening charges $A(x)$ and $\bar{A}(\bar{x})$ parametrise the general solution of the Liouville equation just in the form as was obtained by Liouville [8] (see (A.1)).

From (2.12) we also find the chiral out-field through the chiral $i n$-field and vice versa

$$
\phi_{\text {out }}(x)=\phi_{\text {in }}(x)-\log [\mu A(x)], \quad \phi_{\text {in }}(x)=\phi_{\text {out }}(x)-\log [\mu B(x)] .
$$

These fields have the monodromy $\phi_{\text {in }}(x+2 \pi)=\phi_{\text {in }}(x)+\pi p, \quad \phi_{\text {out }}(x+2 \pi)=\phi_{\text {out }}(x)-\pi p$, and the standard free-field mode expansion

$$
\phi_{\text {in }}(x)=q+\frac{p x}{2}+i \sum_{n \neq 0} \frac{a_{n}}{n} e^{-i n x}, \quad \phi_{\text {out }}(x)=\tilde{q}-\frac{p x}{2}+i \sum_{n \neq 0} \frac{b_{n}}{n} e^{-i n x} .
$$


Thus, the momentum zero modes of the asymptotic fields differ only by their sign, while the transformation of the other modes is highly non-trivial, according to (2.15).

The stress tensor (2.2) obtained from (2.8)-(2.9) is given by the Schwarzian derivative

$$
T(x)=\frac{1}{4} p^{2} \zeta^{\prime 2}(x)+\frac{1}{4}\left(\frac{\zeta^{\prime \prime}(x)}{\zeta^{\prime}(x)}\right)^{2}-\frac{1}{2}\left(\frac{\zeta^{\prime \prime}(x)}{\zeta^{\prime}(x)}\right)^{\prime},
$$

and with (2.12) one finds its 'improved' free-field form in terms of the asymptotic fields

$$
T(x)=\phi_{\text {in }}^{\prime 2}(x)-\phi_{\text {in }}^{\prime \prime}(x)=\phi_{\text {out }}^{\prime 2}(x)-\phi_{\text {out }}^{\prime \prime}(x) .
$$

Hence, the 'improved' free-field stress tensors of the in and out-fields coincide. This will be crucial when we discuss the $S$-matrix.

\section{Canonical structure}

A more transparent free-field interpretation of $\Phi_{\text {in }}$ and $\Phi_{\text {out }}$ is obtained in the Hamiltonian description defined by the first-order action

$$
S[\Phi, \Pi]=\int \mathrm{d} \tau \int_{0}^{2 \pi} \frac{\mathrm{d} \sigma}{2 \pi}\left[\Pi \dot{\Phi}-\left(\frac{1}{2} \Pi^{2}+\frac{1}{2}\left(\partial_{\sigma} \Phi\right)^{2}+2 \mu^{2} e^{2 \Phi}\right)\right],
$$

where $\Phi(\sigma)$ and $\Pi(\sigma)$ are canonically conjugated variables.

The canonical 2-form of Liouville theory induces a symplectic structure on the space of parametrising chiral fields. In appendix A we consider the parameterisation of the general solution by the $i n$-field and obtain $\Omega=\omega+\bar{\omega}$, where $\Omega$ is the canonical 2 -form, $\omega$ is the standard chiral free-field symplectic form for the $i n$-field

$$
\omega=\int_{0}^{2 \pi} \frac{\mathrm{d} x}{2 \pi}\left[\delta \phi_{\mathrm{in}}^{\prime}(x) \wedge \delta \phi_{\mathrm{in}}(x)\right]+\frac{1}{2} \delta p \wedge \delta \phi_{\mathrm{in}}(0),
$$

and $\bar{\omega}$ is its antichiral counterpart. The mode expansion (2.16) then leads to

$$
\Omega=\int_{0}^{2 \pi} \frac{\mathrm{d} \sigma}{2 \pi} \delta \Pi(\tau, \sigma) \wedge \delta \Phi(\tau, \sigma)=\delta p_{\text {in }} \wedge \delta q_{\text {in }}+i \sum_{n \neq 0}\left(\frac{\delta a_{-n} \wedge \delta a_{n}}{n}+\frac{\delta \bar{a}_{-n} \wedge \delta \bar{a}_{n}}{n}\right)
$$

with $q_{\text {in }}=q+\bar{q}$ and $p_{\text {in }}=p$.

The right hand side of (3.3) corresponds to the canonical 2-form of the complete $i n$-field

$$
\Phi_{\text {in }}(\tau, \sigma)=q_{\text {in }}+p_{\text {in }} \tau+i \sum_{n \neq 0}\left(\frac{a_{n}}{n} e^{-i n x}+\frac{\bar{a}_{n}}{n} e^{-i n \bar{x}}\right)
$$

and its inversion provides the Poisson brackets

$$
\left\{p_{\text {in }}, q_{\text {in }}\right\}=1, \quad\left\{a_{m}, a_{n}\right\}=\frac{i}{2} m \delta_{m,-n}, \quad\left\{\bar{a}_{m}, \bar{a}_{n}\right\}=\frac{i}{2} m \delta_{m,-n} .
$$

Thus, the transformation from the $i n$-field to the Liouville field is canonical

$$
\int_{0}^{2 \pi} \frac{\mathrm{d} \sigma}{2 \pi} \delta \Pi(\tau, \sigma) \wedge \delta \Phi(\tau, \sigma)=\int_{0}^{2 \pi} \frac{\mathrm{d} \sigma}{2 \pi} \delta \Pi_{\text {in }}(\tau, \sigma) \wedge \delta \Phi_{\text {in }}(\tau, \sigma) .
$$


The same relation holds for the out-field and, therefore, the map from the in-field to the out-field is canonical as well

$$
\int_{0}^{2 \pi} \frac{\mathrm{d} \sigma}{2 \pi} \delta \Pi_{\text {in }}(\tau, \sigma) \wedge \delta \Phi_{\text {in }}(\tau, \sigma)=\int_{0}^{2 \pi} \frac{\mathrm{d} \sigma}{2 \pi} \delta \Pi_{\text {out }}(\tau, \sigma) \wedge \delta \Phi_{\text {out }}(\tau, \sigma) .
$$

Applying the map (2.15) to the chiral symplectic form (3.2), we find

$$
\omega=\int_{0}^{2 \pi} \frac{\mathrm{d} x}{2 \pi}\left[\delta \phi_{\text {out }}^{\prime}(x) \wedge \delta \phi_{\text {out }}(x)\right]-\frac{1}{2} \delta p \wedge \delta \phi_{\text {out }}(0) .
$$

Hence, the map is canonical in the chiral sectors separately. We will now calculate the corresponding generating functions.

First we consider the generating function $G$ for the canonical transformation in the chiral sector. According to (3.2) and (3.8), it is defined by the differential form

$$
\delta G=\int_{0}^{2 \pi} \frac{\mathrm{d} x}{2 \pi}\left[\phi_{\text {out }}^{\prime}(x) \delta \phi_{\text {out }}(x)-\phi_{\text {in }}^{\prime}(x) \delta \phi_{\text {in }}(x)\right]+\frac{1}{2}\left[\phi_{\text {out }}(0)+\phi_{\text {in }}(0)\right] \delta p .
$$

Using the parameterisation (2.12) and partial integration, we find $G$ as a function of the chiral part of the phase space

$$
G=-p+\frac{\mu}{2} \int_{0}^{2 \pi} \frac{\mathrm{d} x}{2 \pi} \xi^{\prime} \log \xi^{\prime} .
$$

By (2.12) and (2.16), this function can be written in terms of the Fourier modes as follows

$$
G=\frac{1}{2} p(\tilde{q}+q)-p+i \sum_{n \neq 0} \frac{1}{n} a_{-n} b_{n} .
$$

Now we calculate the generating function of the canonical transformation for the complete asymptotic fields. Due to (3.7), the difference between the canonical presymplectic forms of the out and in fields is an exact 1-form

$$
\delta \mathcal{G}\left[\Phi_{\text {out }}, \Phi_{\text {in }}\right]=\int_{0}^{2 \pi} \frac{\mathrm{d} \sigma}{2 \pi}\left[\Pi_{\text {out }}(\tau, \sigma) \delta \Phi_{\text {out }}(\tau, \sigma)-\Pi_{\text {in }}(\tau, \sigma) \delta \Phi_{\text {in }}(\tau, \sigma)\right] .
$$

To find this generating function, we use the parameterisation (2.9), which for the canonical momenta yields

$$
\Pi_{\text {out }}=\frac{1}{2}\left(\frac{\xi^{\prime \prime}}{\xi^{\prime}}+\frac{\bar{\xi}^{\prime \prime}}{\bar{\xi}^{\prime}}\right)-\frac{\mu}{2}\left(\xi^{\prime}+\bar{\xi}^{\prime}\right), \quad \Pi_{\text {in }}=\frac{1}{2}\left(\frac{\xi^{\prime \prime}}{\xi^{\prime}}+\frac{\bar{\xi}^{\prime \prime}}{\bar{\xi}^{\prime}}\right)+\frac{\mu}{2}\left(\xi^{\prime}+\bar{\xi}^{\prime}\right) .
$$

Similarly to (3.9), the integrand in (3.12) can be written as an exact form and one extracts $\mathcal{G}$ as

$$
\mathcal{G}=\frac{\mu}{2} \int_{0}^{2 \pi} \frac{\mathrm{d} \sigma}{2 \pi}\left[\xi^{\prime} \log \xi^{\prime}+\bar{\xi}^{\prime} \log \bar{\xi}^{\prime}-\xi^{\prime} \log \bar{\xi}^{\prime}-\bar{\xi}^{\prime} \log \xi^{\prime}-2\left(\xi^{\prime}+\bar{\xi}^{\prime}\right)\right] .
$$

Note that here $\tau$ is fixed, the arguments of $\xi$ and $\bar{\xi}$ are $x=\tau+\sigma$ and $\bar{x}=\tau-\sigma$, respectively, and ' denotes the derivative with respect to the argument. Hence, $\mathcal{G}$ does not split into the sum of the chiral and antichiral parts and it is $\tau$-dependent. 
From (2.9) follows

$$
\xi^{\prime} \bar{\xi}^{\prime}=e^{\Phi_{+}}, \quad \mu\left(\xi^{\prime}-\bar{\xi}^{\prime}\right)=\partial_{\sigma} \Phi_{-},
$$

with $\Phi_{ \pm}=\Phi_{\text {in }} \pm \Phi_{\text {out }}$, and the generating function (3.14) becomes

$$
\mathcal{G}=\int_{0}^{2 \pi} \frac{\mathrm{d} \sigma}{2 \pi}\left[\frac{1}{2} \partial_{\sigma} \Phi_{-} \log \left(\frac{\sqrt{\left(\partial_{\sigma} \Phi_{-}\right)^{2}+4 \mu^{2} e^{\Phi_{+}}}+\partial_{\sigma} \Phi_{-}}{\sqrt{\left(\partial_{\sigma} \Phi_{-}\right)^{2}+4 \mu^{2} e^{\Phi_{+}}}-\partial_{\sigma} \Phi_{-}}\right)-\sqrt{\left(\partial_{\sigma} \Phi_{-}\right)^{2}+4 \mu^{2} e^{\Phi_{+}}}\right] .
$$

This functional defines the semiclassical $S$-matrix in the $\Phi$-representation. The details of this correspondence are described in the next section.

Here we introduce another generating function $\mathcal{F}$, which is related to the semiclassical $S$-matrix in the Fock space. It is given as a sum of the chiral and antichiral parts $\mathcal{F}=F+\bar{F}$, where $F$ is obtained from the differential form

$$
\delta F=(\tilde{q}+q) \delta p-2 i \sum_{m>0} \frac{1}{m}\left(b_{m} \delta b_{m}^{*}+a_{m}^{*} \delta a_{m}\right),
$$

and one has a similar antichiral 1-form for $\delta \bar{F}$.

Thus, $F$ is treated as a function of the variables $\left(p, b_{m}^{*}, a_{m}\right)$, with $m>0$, and it satisfies the equations

$$
\frac{\partial F}{\partial p}=\tilde{q}+q, \quad \frac{i m}{2} \frac{\partial F}{\partial a_{m}}=a_{m}^{*}, \quad \frac{i m}{2} \frac{\partial F}{\partial b_{m}^{*}}=b_{m} .
$$

The right hand sides can be obtained as functions of $\left(p, b_{m}^{*}, a_{m}\right)$, using the relations between the asymptotic fields (2.15), or (2.18). We will discuss this point further in section 4.1 .

It is not difficult to establish the relation between $F$ and $G$. Indeed, the 1-forms in (3.9) and (3.17) are given as a difference between the presymplectic forms of the asymptotic fields. The difference $F-G$ is, therefore, a quadratic combination of the Fourier modes. Using the mode expansion (2.16) to evaluate (3.9), one finds

$$
F-G=\frac{1}{2} p q_{+}-i \sum_{m>0} \frac{1}{m}\left(b_{m}^{*} b_{m}+a_{m}^{*} a_{m}\right),
$$

with $q_{+}:=\tilde{q}+q$, and from (3.11) follows

$$
F=p q_{+}-p-i \sum_{m>0} \frac{1}{m}\left(b_{m}^{*} b_{m}+a_{m}^{*} a_{m}+b_{m}^{*} a_{m}-a_{m}^{*} b_{m}\right) .
$$

Here the independent variables are, as stated before, $p$ and $a_{m}, b_{m}^{*}$ with $m>0$. We will refer to them as holomorphic variables. For later use we introduce

$$
\alpha_{n}= \begin{cases}a_{n} & n>0 \\ b_{n} & n<0\end{cases}
$$

They do not satisfy any reality condition which would relate $\alpha_{n}$ to $\alpha_{-n}$.

In appendix B we will present another realisation of the generating function $G$. But in our further discussion it is $F(p, \alpha)$ and its Legendre transform which play the central role. 


\section{Semiclassical $S$-matrix}

Assuming that the classical relations

$$
\frac{\delta \mathcal{G}}{\delta \Phi_{\text {out }}(\sigma)}=\frac{1}{2 \pi} \Pi_{\text {out }}(\sigma), \quad \frac{\delta \mathcal{G}}{\delta \Phi_{\text {in }}(\sigma)}=-\frac{1}{2 \pi} \Pi_{\text {in }}(\sigma),
$$

are valid at the quantum level with operator ordering such that $\hat{\Phi}_{\text {out }}$ stands to left of $\hat{\Phi}_{\text {in }}$, one obtains the equations

$$
\begin{aligned}
\frac{1}{2 \pi}\left\langle\Phi_{\text {out }}\left|\hat{\Pi}_{\text {out }}(\sigma)\right| \Phi_{\text {in }}\right\rangle & =-i \hbar \frac{\delta}{\delta \Phi_{\text {out }}(\sigma)}\left\langle\Phi_{\text {out }} \mid \Phi_{\text {in }}\right\rangle=\left\langle\Phi_{\text {out }} \mid \Phi_{\text {in }}\right\rangle \frac{\delta \mathcal{G}}{\delta \Phi_{\text {out }}(\sigma)} \\
\frac{1}{2 \pi}\left\langle\Phi_{\text {out }}\left|\hat{\Pi}_{\text {in }}(\sigma)\right| \Phi_{\text {in }}\right\rangle & =i \hbar \frac{\delta}{\delta \Phi_{\text {in }}(\sigma)}\left\langle\Phi_{\text {out }} \mid \Phi_{\text {in }}\right\rangle=-\left\langle\Phi_{\text {out }} \mid \Phi_{\text {in }}\right\rangle \frac{\delta \mathcal{G}}{\delta \Phi_{\text {in }}(\sigma)}
\end{aligned}
$$

Up to a constant factor, they are solved by

$$
\left\langle\Phi_{\text {out }} \mid \Phi_{\text {in }}\right\rangle=e^{\frac{i}{\hbar} \mathcal{G}\left[\Phi_{\text {out }}, \Phi_{\text {in }}\right]} .
$$

As the assumptions are generally valid up to $\mathcal{O}(\hbar)$ terms, this only holds semiclassically. Due to the complicated form of the functional $\mathcal{G}$ given in (3.16), eq. (4.3) is not convenient for further analysis of the transition amplitudes in Fock space.

We now consider the quantum mechanical treatment of the generating function $F$. The Fourier-mode operators of the in-field satisfy the commutation relations

$$
[\hat{q}, \hat{p}]=i \hbar, \quad\left[\hat{a}_{m}, \hat{a}_{m}^{\dagger}\right]=\frac{1}{2} \hbar m, \quad \text { where } \quad m>0 \quad \text { and } \quad \hat{a}_{m}^{\dagger}=\hat{a}_{-m} .
$$

The $p$-dependent $(p>0)$ vacuum state for the chiral $i n$-field is defined in the standard way as

$$
\hat{p}|p, 0\rangle=p|p, 0\rangle, \quad \hat{a}_{m}|p, 0\rangle=0, \quad \text { for } \quad m>0,
$$

and coherent states are constructed as

$$
|p, a\rangle=\exp \left(\frac{2}{\hbar} \sum_{m>0} \frac{1}{m} a_{m} \hat{a}_{m}^{\dagger}\right)|p, 0\rangle .
$$

The out-field operators and bra-vectors $\left\langle b^{*}, \tilde{p}\right|$, with $\tilde{p}<0$, are defined similarly and one gets

$$
\begin{array}{rlrl}
\hat{a}_{m}|p, a\rangle & =a_{m}|p, a\rangle, & \hat{a}_{m}^{\dagger}|p, a\rangle & =\frac{1}{2} \hbar m \frac{\partial}{\partial a_{m}}|p, a\rangle, \\
\left\langle b^{*}, \tilde{p}\right| \hat{b}_{m}^{\dagger} & =b_{m}^{*}\left\langle b^{*}, \tilde{p}\right|, \quad\left\langle b^{*}, \tilde{p}\right| \hat{b}_{m}=\frac{1}{2} \hbar m \frac{\partial}{\partial b_{m}^{*}}\left\langle b^{*}, \tilde{p}\right| .
\end{array}
$$

To analyze the matrix elements $\left\langle b^{*}, p^{\prime} \mid p, a\right\rangle$, we insert the canonical operators and find

$$
\left\langle b^{*}, \tilde{p}|\hat{\tilde{p}}+\hat{p}| p, a\right\rangle=(\tilde{p}+p)\left\langle b^{*}, \tilde{p} \mid p, a\right\rangle, \quad\left\langle b^{*}, \tilde{p}|\hat{\tilde{q}}+\hat{q}| p, a\right\rangle=i \hbar\left(\partial_{\tilde{p}}-\partial_{p}\right)\left\langle b^{*}, \tilde{p} \mid p, a\right\rangle .
$$

If we require $\hat{\tilde{p}}=-\hat{p}$, which we know to be satisfied by the classical solutions, the left hand side of the first equation vanishes and yields

$$
\left\langle b^{*}, \tilde{p} \mid p, a\right\rangle=\mathcal{S}\left(p, b^{*}, a\right) \delta(p+\tilde{p}) .
$$


Assuming that the classical relations (3.18) are valid at the quantum level, and that the operators $\hat{b}_{n}^{*}$ in $F\left(p, b^{*}, a\right)$ stand to the left of the operators $\hat{a}_{n}$, we obtain from (4.7)-(4.9)

$$
-i \hbar \frac{\partial \mathcal{S}}{\partial p}=\frac{\partial F}{\partial p} \mathcal{S}, \quad \hbar \frac{\partial \mathcal{S}}{\partial a_{m}}=i \frac{\partial F}{\partial a_{m}} \mathcal{S}, \quad \hbar \frac{\partial \mathcal{S}}{\partial b_{m}^{*}}=i \frac{\partial F}{\partial b_{m}^{*}} \mathcal{S}
$$

These equations are similar to (4.2), and we obtain semiclassically

$$
\mathcal{S}_{\mathcal{S}}\left(p, b^{*}, a\right)=e^{\frac{i}{\hbar} F\left(p, b^{*}, a\right)} .
$$

\subsection{Semiclassical amplitudes 1}

Our goal is to find the generating function $F$ in terms of the holomorphic variables, i.e. $F\left(p, b^{*}, a\right)$. To construct this function from eqs. (3.18), one has to express their right hand sides as functions of $\left(p, b^{*}, a\right)$, using the relations between the $i n$ - and out-fields, and then integrate. The relation between $\left(q_{+}, b, a^{*}\right)$ and $\left(p, b^{*}, a\right)$ is encoded in

$$
\phi_{\text {in }}(x)+\phi_{\text {out }}(x)=\log \left[\phi_{\text {in }}^{\prime}(x)-\phi_{\text {out }}^{\prime}(x)\right]-\log \mu,
$$

which follows from (2.12). This equation contains the full dynamical content of classical scattering of Liouville theory on the cylinder and we can use it to compute $F$, as we will now demonstrate. The reason for such a simple relation to exist is the classical integrability of the theory.

Expanded in Fourier modes it yields

$$
q+\tilde{q}+i \sum_{n \neq 0} \frac{a_{n}+b_{n}}{n} e^{-i n x}=\log (p / \mu)+\log \left(1+\sum_{n \neq 0} \frac{a_{n}-b_{n}}{p} e^{-i n x}\right),
$$

from which we find the relations

$$
\begin{aligned}
q+\tilde{q} & =\log (p / \mu)-\frac{1}{p^{2}} \sum_{m>0}\left(a_{m}^{*}-b_{m}^{*}\right)\left(a_{m}-b_{m}\right)+\ldots, \\
i \frac{a_{n}+b_{n}}{n} & =\frac{a_{n}-b_{n}}{p}+\ldots .
\end{aligned}
$$

The modes $b_{m}, a_{m}^{*}$, for $m>0$, and $q+\tilde{q}$ can be obtained from these relations in power series of the holomorphic variables $\left(b^{*}, a\right)$ with $p$-dependent coefficients

$$
\begin{aligned}
& b_{m}=\frac{m-i p}{m+i p} a_{m}+\ldots, \quad a_{m}^{*}=\frac{m-i p}{m+i p} b_{m}^{*}+\ldots, \\
& q+\tilde{q}=\log (p / \mu)-\sum_{m>0} \frac{4 b_{m}^{*} a_{m}}{(m+i p)^{2}}+\ldots .
\end{aligned}
$$

Integration of (3.18) gives $F\left(p, b^{*}, a\right)=F^{(0)}+F^{(2)}+F^{(3)}+\cdots$, where the upper index indicates the total degree of the holomorphic variables. From (4.15) we then have

$$
F^{(0)}=p[\log (p / \mu)-1], \quad F^{(2)}=-2 i \sum_{m>0} \frac{1}{m}\left(\frac{m-i p}{m+i p}\right) b_{m}^{*} a_{m} .
$$


The term $F^{(0)}(p)$, given in (4.16), defines the semiclassical reflection amplitude

$$
R_{0}(p)=e^{\frac{2 i}{\hbar} F^{(0)}}
$$

of the $p$-dependent vacuum state $|p, 0\rangle$. The total reflection amplitude contains the same contribution from the antichiral part. This doubling is taken into account in (4.17), which is common for all transition amplitudes. The higher level states have additional contributions. For instance, from the expression for $F^{(2)}$ we see that the scattering of the $i n$-state $\hat{a}_{m}^{\dagger}|p, 0\rangle$ is given by the additional phase factor $\frac{m-i p}{m+i p}$.

The calculation of $F^{(3)}$ requires the next order terms in (4.14)-(4.15), and the continuation of this procedure defines $F\left(p, b^{*}, a\right)$ as a power series in holomorphic variables $\left(b^{*}, a\right)$ with $p$-dependent coefficients.

In the following we will present two alternatives to obtain the $F^{(\nu)}$, both of which are more efficient and they can be generalised to obtain the quantum scattering amplitudes.

\subsection{Relation to classical one-dimensional field theory}

We will now use the relation between the in- and out-fields to construct the Legendre transform of $F$, or rather of a slightly modified version of $F$, which we will call $\tilde{F}$; it will be defined momentarily. As a consequence of the general relation between semiclassical scattering amplitudes and tree-level Feynman diagrams [10] we expect that the Legendre transform of $\tilde{F}$, which we will call $S$, is the action from which the Feynman rules are derived and the tree-level Feynman diagrams generate $\tilde{F}$ and, therefore, $F$. In the next section we will show that the quantum corrections to the scattering amplitudes are contained in the loop diagrams derived from $S$.

To proceed, we first rewrite eq. (4.12) in the form

$$
\phi_{\mathrm{in}}^{\prime}-\phi_{\mathrm{out}}^{\prime}=\mu e^{\phi_{\mathrm{in}}+\phi_{\mathrm{out}}} .
$$

We express this in terms of the modes

$$
p+\sum_{n \neq 0} \beta_{n} e^{-i n x}=\mu e^{q+} e^{i \sum_{n \neq 0} \frac{1}{n} \gamma_{n} e^{-i n x}},
$$

where we have defined the combinations

$$
\gamma_{n}=a_{n}+b_{n}, \quad \beta_{n}=a_{n}-b_{n} .
$$

They are related to $\alpha_{n}$, which were defined in (3.21) via

$$
\alpha_{n}=\frac{1}{2} \gamma_{n}+\frac{1}{2} \epsilon(n) \beta_{n} .
$$

Here $\epsilon(n)$ is the sign-function, i.e. $\epsilon(n)=+1$ for $n>0$ and $\epsilon(n)=-1$ for $n<0$. It will turn out to be convenient to rescale the modes as

$$
\alpha_{n}=-\frac{i \epsilon(n)}{2} j_{n}
$$


with

$$
j_{n}=i \epsilon(n) \gamma_{n}+i \beta_{n}
$$

If we define ${ }^{1}$

$$
\tilde{F}=F-F^{(0)}-i \sum_{n \neq 0} \frac{\alpha_{n} \alpha_{-n}}{|n|}=F-p\left(\ln \frac{p}{\mu}-1\right)-2 i \sum_{m>0} \frac{1}{m} b_{m}^{*} a_{m},
$$

we find

$$
\frac{\partial \tilde{F}}{\partial j_{-n}}=\frac{1}{n} \gamma_{n} \equiv-i \varphi_{n}
$$

Note that while the $\alpha_{n}$ and therefore also the $j_{n}$ did not satisfy any reality condition, $\varphi_{n}^{*}=\varphi_{-n}$, i.e. they are the Fourier modes of the real field $\varphi=\phi_{\text {in }}+\phi_{\text {out }}$.

If we now use

$$
\beta_{n}=\mu e^{q_{+}} \int_{0}^{2 \pi} e^{i n x} e^{i \sum_{k \neq 0} \frac{1}{k} \gamma_{k} e^{-i k x}}=\mu e^{q_{+}} \int_{0}^{2 \pi} \frac{d x}{2 \pi} e^{i n x} e^{\sum_{k \neq 0} \varphi_{k} e^{-i k x}},
$$

which follows from (4.19), we can write (4.23) in the form

$$
j_{n}=|n| \varphi_{n}+i \mu e^{q_{+}} \int_{0}^{2 \pi} \frac{d x}{2 \pi} e^{i n x} e^{\sum_{k \neq 0} \varphi_{k} e^{-i k x}} \stackrel{!}{=} \frac{\partial S}{\partial \varphi_{-n}},
$$

where $S$ is defined to be the Legendre transform of $\tilde{F}$.

While $j_{0}$ has not yet been defined, the above relation has an obvious extension to $n=0$ with $j_{0}=i p$. Here we have used

$$
p=\mu e^{q_{+}} \int_{0}^{2 \pi} \frac{d x}{2 \pi} e^{i \sum_{k \neq 0} \frac{1}{k} \gamma_{k} e^{-i k x}},
$$

which is the zero mode of (4.19). It is now also natural to extend (4.25) to $n=0$ and to define

$$
\tilde{q}_{+} \equiv \varphi_{0}=\frac{\partial \tilde{F}}{\partial p}=q_{+}-\ln \frac{p}{\mu}
$$

such that $\mu e^{q_{+}}=p e^{\tilde{q}_{+}}=p e^{\varphi_{0}}$.

We now integrate (4.27) and find

$$
S=\frac{1}{2} \sum_{n \in \mathbb{Z}} \varphi_{-n}|n| \varphi_{n}+i p \int_{0}^{2 \pi} \frac{d x}{2 \pi} e^{\sum_{k \in \mathbb{Z}} \varphi_{k} e^{-i k x}} .
$$

This finally gives a non-local action with exponential potential and an imaginary coupling constant

$$
S=\int_{0}^{2 \pi} \frac{d x}{2 \pi}\left(\frac{1}{2} \varphi(x) \sqrt{-\partial_{x}^{2}} \varphi(x)+i p e^{\varphi(x)}\right)
$$

when written in 'position space'. Note that in this expression $p$ appears as a coupling constant. It is not a dynamical variable. Those are $\varphi_{n}, n \in \mathbb{Z}$.

\footnotetext{
${ }^{1}$ Even though we have no compelling a priori reason for this definition, we want to remark that the terms which are subtracted from $F$ to obtain $\tilde{F}$ are those terms in $F$ which survive in the limit $p \rightarrow \infty$, while keeping all other parameters, including the mode numbers, finite.
} 
To recapitulate, we have introduced the conjugated pairs $\left\{j_{n}, \varphi_{-n}\right\}$ and the two functions $\{\tilde{F}(j), S(\varphi)\}$ which are Legendre transformed of each other

$$
S(\varphi)=\langle\varphi j\rangle-i \tilde{F}(j),
$$

where

$$
\langle\varphi j\rangle=\int_{0}^{2 \pi} \frac{d x}{2 \pi} j(x) \varphi(x)=\sum_{n \in \mathbb{Z}} j_{n} \varphi_{-n} .
$$

In other words,

$$
e^{\frac{i}{\hbar} \tilde{F}(j)}=\left.\int D \varphi e^{-\frac{1}{\hbar}(S(\varphi)-\langle\varphi j\rangle)}\right|_{\frac{\delta S}{\delta \varphi}=j},
$$

where the functional integral over $\varphi$ is evaluated at the saddle point. In the next section we will include quantum corrections, i.e. fluctuations around the saddle point.

As mentioned before, $\tilde{F}$ is the generating functional for the tree level graphs computed with $S$ or, more precisely, due to the relation (4.11), of the connected graphs. We will now compute the lowest order $n$-point functions and will then verify them by an explicit calculation using (2.18).

The Feynman rules derived from (4.31) are: ${ }^{2}$

1. Draw all connected $\nu$-point tree diagrams with $r$-valent vertices for all $r \geq 3$. Each external line carries an index $n_{i}, i=1, \ldots, \nu$. The labels of the internal lines are dictated by 'energy' conservation at each vertex.

2. To each $r$-valent vertex assign a factor $-i p$.

3. To each line, including the external ones, assign a propagator $\frac{1}{|n|+i p}$ where $n$ is the 'energy' carried by the line. All external lines are ingoing.

4. Sum over all distinct diagrams.

Note that in rule 3 . we have included the quadratic term of the potential into the propagator.

We now illustrate these rules, noting that the external lines always have $n_{i} \neq 0$, as they correspond to oscillator excitations. This excludes tadpoles. There is no such restriction for the internal lines.

We expand ${ }^{3}$

$$
\tilde{F}=\sum_{\nu=2}^{\infty} \frac{1}{\nu !} \sum_{n_{i} \neq 0} \tilde{f}_{n_{1}, \ldots, n_{\nu}}^{(\nu)} j_{n_{1}} \cdots j_{n_{\nu}} \delta_{n_{1}+\cdots+n_{\nu}},
$$

and write the amplitudes for $\nu \geq 3$ in the form

$$
i \tilde{f}_{n_{1} \ldots n_{\nu}}^{(\nu)}=-i p\left(\prod_{j=1}^{\nu} \frac{1}{\left|n_{j}\right|+i p}\right) U_{n_{1} \ldots n_{\nu}}^{(\nu)},
$$

\footnotetext{
${ }^{2}$ Here we set $\hbar=1$.

${ }^{3}$ For $\nu \geq 3$ the expansions for $F$ and $\tilde{F}$ coincide.
} 
where we have factored out the contributions from the external lines and one power of the coupling constant, i.e. the $\nu$-point vertex contribution. We will also use $i \tilde{f}^{(2)}=\frac{1}{|n|+i p}$. Therefore

$$
i F^{(2)}=i \sum_{n \neq 0} \frac{1}{2} \tilde{f}^{(2)} j_{n} j_{-n}-\sum_{n \neq 0} \frac{1}{|n|} \alpha_{n} \alpha_{-n}=\sum_{n \neq 0} \frac{1}{|n|}\left(\frac{|n|-i p}{|n|+i p}\right) \alpha_{n} \alpha_{-n},
$$

which agrees with (4.16).

We will now evaluate a few tree-level diagrams. Loop diagrams will be considered in section 5 .

The simplest diagram is the three-point function: there is only the three-point vertex contribution

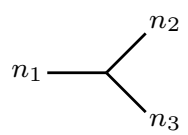

According to the Feynman rules, it evaluates to

$$
-i p \prod_{i=1}^{3} \frac{1}{\left|n_{i}\right|+i p}
$$

and therefore $U^{(3)}=1$.

For the four-point function there are four distinct diagrams: a direct four-point vertex and three exchange diagrams $(s, t, u$-channels in the language of particle physics):

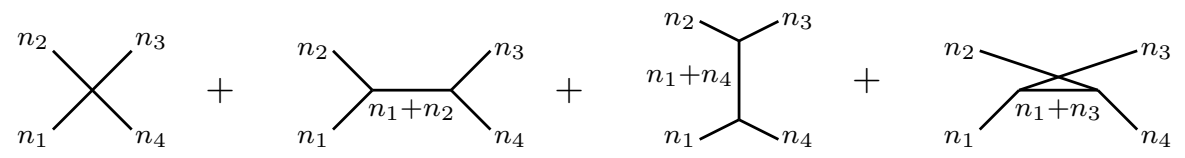

The first diagram evaluates to

$$
-i p \prod_{i=1}^{4} \frac{1}{\left|n_{i}\right|+i p}
$$

while the second, the $s$-channel diagram, becomes

$$
(-i p)^{2}\left(\prod_{i=1}^{4} \frac{1}{\left|n_{i}\right|+i p}\right) \cdot \frac{1}{\left|n_{1}+n_{2}\right|+i p} .
$$

If we sum over all four diagrams we obtain

$$
U_{n_{1} n_{2} n_{3} n_{4}}=1-\frac{i p}{\left|n_{1}+n_{2}\right|+i p}-\frac{i p}{\left|n_{1}+n_{3}\right|+i p}-\frac{i p}{\left|n_{1}+n_{4}\right|+i p} .
$$

As a last example we consider the five-point function. There are now three topologically distinct diagrams:
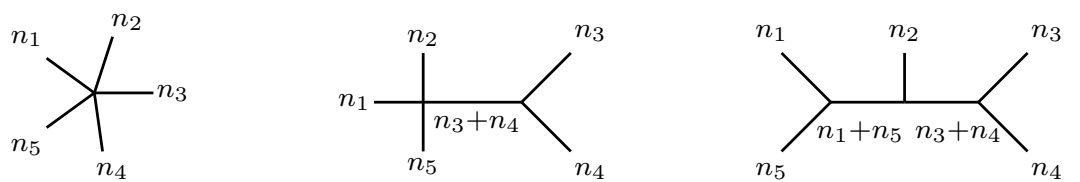
For a given 'energy' assignment of the external lines, there are $\left(\begin{array}{l}5 \\ 2\end{array}\right)=10$ distinct channels with the topology of the second diagram and $\frac{1}{2}\left(\begin{array}{l}5 \\ 2\end{array}\right)\left(\begin{array}{l}3 \\ 2\end{array}\right)=15$ for the third diagram. The first diagram gives

$$
-i p \prod_{i=1}^{5} \frac{1}{\left|n_{i}\right|+i p}
$$

The second diagram evaluates to

$$
-i p\left(\prod_{i=1}^{5} \frac{1}{\left|n_{i}\right|+i p}\right) \cdot \frac{-i p}{\left|n_{3}+n_{4}\right|+i p}
$$

and the third diagram to

$$
-i p\left(\prod_{i=1}^{5} \frac{1}{\left|n_{i}\right|+i p}\right) \cdot \frac{-i p}{\left|n_{1}+n_{5}\right|+i p} \cdot \frac{-i p}{\left|n_{3}+n_{4}\right|+i p} .
$$

Summing over all diagrams we find

$$
U^{(5)}=1+\sum_{i<j} \frac{(-i p)}{\left|n_{i}+n_{j}\right|+i p}+\sum_{\{i<j\} \cap\{k<l\}=0} \frac{(-i p)}{\left|n_{i}+n_{j}\right|+i p} \cdot \frac{(-i p)}{\left|n_{k}+n_{l}\right|+i p}
$$

and so on for the higher $U^{(\nu)}$.

\subsection{Semiclassical amplitudes 2}

We will now compute $F$ directly. In section 4.1 we have shown how to use (4.12) and the relations (3.18) to compute $F$. In this way we have obtained $F^{(0)}$ and $F^{(2)}$ and in principle one can continue to higher orders. A more efficient algorithm for finding higher order terms of $F$ can be extracted from relation (2.18), which equates the free-field improved energy momentum tensors expressed through the asymptotic in- and out-fields. With the mode-expansions (2.16) and

$$
T(x)=\sum_{n \in \mathbb{Z}} L_{n} e^{-i n x},
$$

one obtains $L_{n}$ 's in terms of the Fourier modes of the $i n$-field

$$
L_{0}=\frac{1}{4} p^{2}+\sum_{k \neq 0} a_{-k} a_{k}, \quad L_{n}=(p+i n) a_{n}+\sum_{k, l \neq 0} a_{k} a_{l} \delta_{k+l, n}, \quad(n \neq 0) .
$$

The out-field mode expansion of $L_{n}$ 's is obtained by replacing $p \mapsto-p, a_{n} \mapsto b_{n}$.

Using complex conjugation and only positive indices for the Fourier modes of the asymptotic fields, the equality between $L_{n}$ 's of the in and out-fields can be written in the form

$$
\begin{aligned}
\sum_{j \geq 1} a_{j}^{*} a_{j} & =\sum_{j \geq 1} b_{j}^{*} b_{j} \\
(p+i m) a_{m}+(p-i m) b_{m} & =\sum_{j, j^{\prime} \geq 1}\left[\left(b_{j} b_{j^{\prime}}-a_{j} a_{j^{\prime}}\right) \delta_{j+j^{\prime}, m}+2\left(b_{j} b_{j^{\prime}}^{*}-a_{j} a_{j^{\prime}}^{*}\right) \delta_{j-j^{\prime}, m}\right], \\
(p-i m) a_{m}^{*}+(p+i m) b_{m}^{*} & =\sum_{j, j^{\prime} \geq 1}\left[\left(b_{j}^{*} b_{j^{\prime}}^{*}-a_{j}^{*} a_{j^{\prime}}^{*}\right) \delta_{j+j^{\prime}, m}+2\left(b_{j}^{*} b_{j^{\prime}}-a_{j}^{*} a_{j^{\prime}}\right) \delta_{j-j^{\prime}, m}\right],
\end{aligned}
$$


where $m>0$. These relations become equations for $\left(b, a^{*}\right)$. They have solutions as power series in the holomorphic variables $\left(b^{*}, a\right)$ with $p$-dependent coefficients. The function $F\left(p, b^{*}, a\right)$ is then obtained by integration of (3.18). This leaves, however, $F^{(0)}$ undetermined.

Alternatively, replacing the variables $\left(b, a^{*}\right)$ in (4.51) by the derivatives of $F$, according to (3.18), we find differential equations directly for the function $F$ which can be solved as power series in the holomorphic variables. This turns out to be the more efficient procedure. In order to get a covariant structure for scattering amplitudes, it is convenient to use instead of the modes $a_{m}$ and $b_{m}^{*}$ for $m>0$, the modes $\alpha_{n}$ which we defined in (3.21) for all integer $n \neq 0$. Doing this we find from (4.51) the system of first order non-linear differential equations for $F(p, \alpha)$

$$
\sum_{k \neq 0} k \alpha_{k} \frac{\partial F}{\partial \alpha_{k}}=0
$$

and

$$
\begin{aligned}
|n|(|n|+i p) \frac{\partial F}{\partial \alpha_{n}}+2 i & (|n|-i p) \alpha_{-n}+i \sum_{k, l \neq 0}[\epsilon(k)-\epsilon(l)]|l| \alpha_{k} \frac{\partial F}{\partial \alpha_{-l}} \delta_{k+l+n} \\
& +\sum_{k, l \neq 0}[\epsilon(k)+\epsilon(l)]\left(\alpha_{k} \alpha_{l}+\frac{1}{4}|k||l| \frac{\partial F}{\partial \alpha_{-k}} \frac{\partial F}{\partial \alpha_{-l}}\right) \delta_{k+l+n}=0,
\end{aligned}
$$

where $n$ is a non-zero integer.

A monomial $\alpha_{n_{1}} \cdots \alpha_{n_{\nu}}$ solves the linear equation (4.52) if $n_{1}+\cdots+n_{\nu}=0$ and the function $F$ is then represented as a power series; cf. (4.24) and (4.35).

At this point it turns out to be convenient to introduce $\tilde{F}$ and the $j_{n}$ of section 4.2 . They lead to the simpler equation for $\tilde{F}$ :

$$
i n(|n|+i p) \frac{\partial \tilde{F}}{\partial j_{n}}-n j_{-n}-i \sum_{k, l \neq 0} l j_{k} \frac{\partial \tilde{F}}{\partial j_{-l}} \delta_{k+l+n}-\sum_{k, l \neq 0}|k| l \frac{\partial \tilde{F}}{\partial j_{-k}} \frac{\partial \tilde{F}}{\partial j_{-l}} \delta_{k+l+n}=0 .
$$

We insert the Ansatz (4.35) and solve (4.54) order by order in the $\alpha_{n}$ and determine the amplitudes $f^{(\nu)}$ recursively.

At the linear order, we find

$$
i \tilde{f}^{(2)}=\frac{1}{|n|+i p}
$$

and at the quadratic order

$$
i \tilde{f}^{(3)}=-i p \cdot \prod_{i=1}^{3} \frac{1}{\left|n_{i}\right|+i p} .
$$

Both are in agreement with the results presented in section 4.2. The first non-trivial case comes from solving (4.54) at cubic order. After a somewhat tedious calculation one finds again complete agreement with the Feynman diagram calculation in section 4.2, i.e. with (4.43).

Since the relation (2.18), which we exploited to obtain the differential equation obeyed by $F$, follows immediately from (4.12) by differentiating w.r.t. $x$, it should be clear that 
we can also find $S$ starting from (4.54). Indeed, using the relations

$$
j_{n}=\frac{\partial S}{\partial \varphi_{-n}}, \quad \varphi_{n}=i \frac{\partial \tilde{F}}{\partial j_{-n}} \quad(n \neq 0)
$$

in (4.54), it becomes

$$
n(|n|+i p) \varphi_{-n}-n \frac{\partial S}{\partial \varphi_{n}}-\sum_{k, l \neq 0} l \varphi_{l} \frac{\partial S}{\partial \varphi_{-k}} \delta_{k+l+n}+\sum_{k, l \neq 0}|k| l \varphi_{k} \varphi_{l} \delta_{k+l+n}=0 .
$$

The simplification effected by passing from $F$ to the shifted $\tilde{F}$ is that the quadratic terms in the modes have cancelled in (4.54) and the functional differential equation satisfied by $S$ is therefore linear. If we make the Ansatz

$$
S=\frac{1}{2} \sum_{n \in \mathbb{Z}}|n| \varphi_{-n} \varphi_{n}+\int_{0}^{2 \pi} \frac{d x}{2 p i} V(\varphi)
$$

we obtain a simple equation for $V$ :

$$
-i p n \varphi_{-n}+n \frac{\partial V}{\partial \varphi_{n}}+\sum_{k, l \neq 0} l \varphi_{l} \frac{\partial V}{\partial \varphi_{-k}} \delta_{k+l+n}=0 .
$$

Here a zero mode for $\varphi$ is not included. But we can define $\varphi_{0}$ as the variable conjugate to $j_{0}=\frac{\partial V}{\partial \varphi_{0}}$. If we identify the latter with $i p$, we find $\varphi_{0}=i \frac{\partial \tilde{F}}{\partial j_{0}}=\tilde{q}_{+}$, as in the discussion in section 4.1. The equation for $V$ is then solved by $V(\varphi)=i p e^{\varphi}$. The constant term in $V$ stays undetermined by eq. (4.60), but it can be chosen arbitrarily, as it does not affect the amplitudes. Here it is chosen as $i p$.

\section{Quantum $S$-matrix}

After having computed the semiclassical scattering amplitudes, we now turn to the quantum corrected amplitudes. We compute them in two alternative ways. We first use the classical action $S$, from which, as we have just shown, the semiclassical amplitudes can be obtained as tree-level Feynman diagrams, to compute loop amplitudes. We then generalise the procedure of section 4.3 by equating the quantum-corrected energy-moment tensors for the asymptotic in- and out-fields. We then compare and find agreement.

The second method is computationally superior and it produces results which are valid to all orders in $\hbar$, in agreement with an old result of Zamolodchikov and Zamolodchikov [6]. We will see that the simplicity of the equation satisfied by $\tilde{F}$ is destroyed and we have not been able to use it to get the full quantum effective action, i.e. the generating functional for the quantum scattering amplitudes, by integrating this equation, as we did in section 4.3.

\subsection{Quantum amplitudes 1}

The starting point is the action (4.30). The Feynman rules are the same as before, but now we allow loops with the only restriction that self-contractions of two lines emanating from the same vertex are not allowed. This corresponds to 'normal ordering' of the potential. It 
eliminates all possible divergences and therefore no further renormalisation is necessary. ${ }^{4}$ We also have to take into account symmetry factors, just as in conventional scalar field theory. We now evaluate a few one-loop diagrams.

For the one-loop contribution of the two-point function there is a single diagram ${ }^{5}$

$$
i \tilde{f}_{-n n}^{(2,1)}=n-\bigcirc-n=\frac{1}{2}(-i p)^{2} \Delta(n)^{2} \sum_{m=-\infty}^{\infty} \Delta(m) \Delta(m+n) .
$$

We have defined the propagator

$$
\Delta(n)=\frac{1}{|n|+i p},
$$

and we have included a factor $-i p$ for each vertex; $1 / 2$ is the appropriate symmetry factor. The (convergent) sum can be computed for general $n$ in terms of the digamma function $\psi$, but the expression is not very illuminating and we will not write it. A few special cases are

$$
\tilde{f}_{-11}^{(2,1)}=i p \Delta(1)^{2}, \quad \tilde{f}_{-22}^{(2,1)}=-\frac{1}{2} i p\left(2 p^{2}-4 i p-1\right) \Delta(1)^{2} \Delta(2)^{2} .
$$

Next we look at one-loop diagrams with three external legs. There are three possible topologies

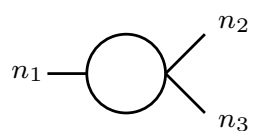

$$
D_{1}^{(3,1)}\left(n_{1}, n_{2}, n_{3}\right)
$$

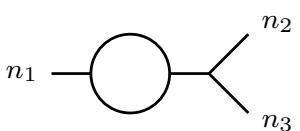

$$
D_{2}^{(3,1)}\left(n_{1}, n_{2}, n_{3}\right)
$$

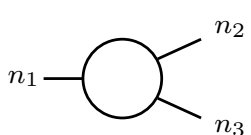

$$
D_{3}^{(3,1)}\left(n_{1}, n_{2}, n_{3}\right)
$$

and the amplitude is

$$
\begin{aligned}
i f_{n_{1} n_{2} n_{3}}^{(3,1)}= & \frac{1}{2}\left(D_{1}^{(3,1)}\left(n_{1}, n_{2}, n_{3}\right)+D_{1}^{(3,1)}\left(n_{2}, n_{3}, n_{1}\right)+D_{1}^{(3,1)}\left(n_{3}, n_{1}, n_{2}\right)\right. \\
& \left.+D_{2}^{(3,1)}\left(n_{1}, n_{2}, n_{3}\right)+D_{2}^{(3,1)}\left(n_{2}, n_{3}, n_{1}\right)+D_{2}^{(3,1)}\left(n_{3}, n_{1}, n_{2}\right)\right) \\
& +D_{3}^{(3,1)}\left(n_{1}, n_{2}, n_{3}\right) .
\end{aligned}
$$

All lines are ingoing with $n_{1}+n_{2}+n_{3}=0$. Using the Feynman rules it is straightforward to evaluate the diagrams; for example

$$
n_{1}-\mathcal{-}_{n_{3}}^{n_{2}}=(-i p)^{3} \prod_{i=1}^{3} \Delta\left(n_{i}\right) \sum_{n=-\infty}^{\infty} \Delta(n) \Delta\left(n+n_{1}\right) \Delta\left(n+n_{1}+n_{2}\right) .
$$

The sum can be performed for any given $n_{1}, n_{2}, n_{3}$ with $\sum n_{i}=0 .{ }^{6}$ Some examples of the complete amplitudes are

$$
\begin{aligned}
& i \tilde{f}_{-211}^{(3,1)}=i p\left(p^{2}+i p+5\right) \Delta(1)^{3} \Delta(2)^{2}, \\
& i \tilde{f}_{-312}^{(3,1)}=-i p\left(p^{4}-i p^{3}+14 p^{2}-29 i p-11\right) \Delta(1)^{3} \Delta(2)^{2} \Delta(3)^{2} .
\end{aligned}
$$

\footnotetext{
${ }^{4}$ We have not proven this statement but the overall degree of divergence of every diagram is clearly negative.

${ }^{5}$ Here we use the notation $f^{(n, \ell)}$ for the $\ell$-loop contribution for the $n$-point function.

${ }^{6}$ E.g. with Mathematica. One can also work out a general expression for generic $n_{i}$ in terms of Polygamma functions, but it is rather lengthy and does not seem to be particularly useful.
} 
For four external lines there are the following ten topologies:

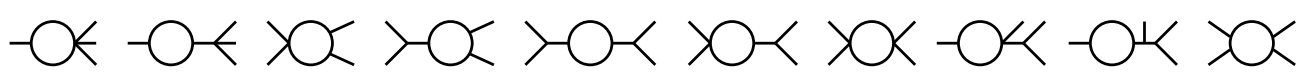

Using the Feynman rules it is again straightforward to evaluate the diagrams and to add them. A few examples for complete one-loop four-point amplitudes are

$$
\begin{aligned}
i \tilde{f}_{-1-111}^{(4,1)} & =-2 i p\left(p^{2}+2 i p+7\right) \Delta(2)^{2} \Delta(1)^{4} \\
i \tilde{f}_{-3111}^{(4,1)} & =2 i p\left(p^{4}+4 i p^{3}+30 p^{2}-20 i p+29\right) \Delta(1)^{4} \Delta(2)^{2} \Delta(3)^{2}, \\
i \tilde{f}_{-2-112}^{(4,1)} & =2 i p\left(p^{4}+2 i p^{3}+25 p^{2}-30 i p+6\right) \Delta(1)^{3} \Delta(2)^{2} \Delta(3)^{2} .
\end{aligned}
$$

It is easy to work out others, but the order of the polynomial in $p$ in the numerator grows fast with the occupation numbers of the external lines.

In principle one can go higher in the loop expansion, but the number of diagrams grows quickly and the multiple sums can no longer be performed in closed form. For instance, the following diagrams contribute to the two-loop corrections of the propagator

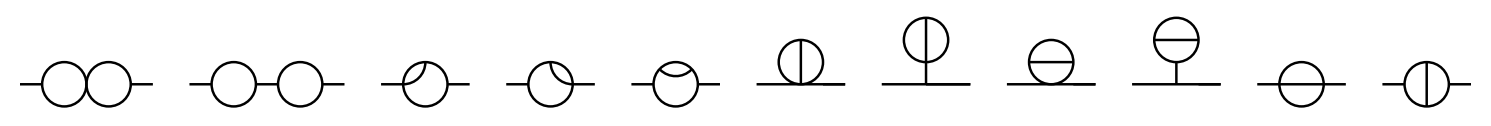

The double sums seem hard to do analytically, but one can do them numerically for some fixed values of $p$ (they converge rather slowly). This and the one-loop results can be compared with the expressions obtained by the second method that we have alluded to and to which we now turn.

\subsection{Quantum amplitudes 2}

A consistent quantization of Liouville theory leads to a deformation of the improvement term in the stress tensor [13-15]. The quantum Virasoro generators in terms of the in-field variables read (cf. (4.50))

$$
\begin{aligned}
\hat{L}_{0} & =\frac{1}{4}\left(\hat{p}^{2}+\eta^{2}\right)+2 \sum_{j \geq 1} \hat{a}_{j}^{\dagger} \hat{a}_{j}, \\
\hat{L}_{m} & =(\hat{p}+i m \eta) \hat{a}_{m}+\sum_{j, j^{\prime} \geq 1} \hat{a}_{j} \hat{a}_{j^{\prime}} \delta_{j+j^{\prime}, m}+2 \sum_{j>0} \hat{a}_{j}^{\dagger} \hat{a}_{m+j}, \\
\hat{L}_{-m} & =(\hat{p}-i m \eta) \hat{a}_{m}^{\dagger}+\sum_{j, j^{\prime} \geq 1} \hat{a}_{j}^{\dagger} \hat{a}_{j^{\prime}}^{\dagger} \delta_{j+j^{\prime}, m}+2 \sum_{j \geq 1} \hat{a}_{m+j}^{\dagger} \hat{a}_{j},
\end{aligned}
$$

where $m \geq 1$ and $\eta=1+\hbar$. The same generators in terms of the out-field variables are obtained by the replacements $p \mapsto-p, \hat{a}_{j} \mapsto \hat{b}_{j}$ and $\hat{a}_{j}^{\dagger} \mapsto \hat{b}_{j}^{\dagger}$.

The zero mode operators fix the transition amplitude between the in and out coherent states (4.6) as in (4.9), where the freedom is given by the function $\mathcal{S}\left(p, b^{*}, a\right)$. Inserting the Virasoro generators between the coherent states $\left\langle\tilde{p}, b^{*}\left|\hat{L}_{n}\right| p, a\right\rangle$ and using the relations (4.7), one obtains equations for the function $\mathcal{S}\left(p, b^{*}, a\right)$. 
To get a covariant structure of the scattering amplitudes we again combine the positive and negative indices of $L_{n}$ 's, as we did in the semiclassical treatment. We then obtain the following equations

$$
\begin{aligned}
& \sum_{k \neq 0} k \alpha_{k} \frac{\partial \mathcal{S}}{\partial \alpha_{k}}=0 \\
& \hbar|n|(p-i|n| \eta) \frac{\partial \mathcal{S}}{\partial \alpha_{n}}+2(p+i|n| \eta) \alpha_{-n} \mathcal{S} \\
& \quad+\hbar \sum_{k, l \neq 0}[\epsilon(k)-\epsilon(l)]|l| \alpha_{k} \frac{\partial \mathcal{S}}{\partial \alpha_{-l}} \delta_{k+l+n} \\
& \quad+\sum_{k, l \neq 0}[\epsilon(k)+\epsilon(l)]\left(\alpha_{k} \alpha_{l} \mathcal{S}-\hbar^{2} \frac{|k||l|}{4} \frac{\partial^{2} \mathcal{S}}{\partial \alpha_{-k} \partial \alpha_{-l}}\right) \delta_{k+l+n}=0 .
\end{aligned}
$$

We represent $\mathcal{S}$ in the form ${ }^{7}$

$$
\mathcal{S}=R(p) e^{\frac{i}{\hbar} F_{q}}
$$

where $R(p)$ is interpreted as the reflection amplitude, which is known as the 2-point function of Liouville theory $[6,11,12]$

$$
R(p)=-\left(\mu^{2} \frac{\sin (\pi \hbar)}{\pi \hbar} \Gamma^{2}(\hbar)\right)^{-\frac{i p}{\hbar}} \frac{\Gamma(i p / \hbar)}{\Gamma(-i p / \hbar)} \frac{\Gamma(i p)}{\Gamma(-i p)} .
$$

It reduces to (4.17) in the $\hbar \rightarrow 0$ limit. This factor is canceled in (5.11)-(5.12) and $F_{q}$ satisfies

$$
\begin{aligned}
|n|(|n| & +i p) \frac{\partial F_{q}}{\partial \alpha_{n}}+2 i(|n| \eta-i p) \alpha_{-n} \\
& +i \sum_{k, l \neq 0}[\epsilon(k)-\epsilon(l)]|l| \alpha_{k} \frac{\partial F_{q}}{\partial \alpha_{-l}} \delta_{k+l+n} \\
& +\sum_{k, l \neq 0}[\epsilon(k)+\epsilon(l)]\left[\alpha_{k} \alpha_{l}+\frac{|k||l|}{4}\left(\frac{\partial F_{q}}{\partial \alpha_{-k}} \frac{\partial F_{q}}{\partial \alpha_{-l}}-i \hbar \frac{\partial^{2} F_{q}}{\partial \alpha_{-k} \partial \alpha_{-l}}\right)\right] \delta_{k+l+n}=0
\end{aligned}
$$

which is the quantum analogue of (4.53). Planck's constant appears through $\eta$ and in the last term. It is this last term which makes the solution of this equation considerably more difficult than in the semiclassical case.

If we again introduce $\tilde{F}$ as

$$
\tilde{F}_{q}=F_{q}-i \sum_{n \neq 0} \frac{\alpha_{n} \alpha_{-n}}{|n|}
$$

and perform the change of variables (4.22), as we did in the semiclassical discussion, we arrive at the equation

$$
\begin{aligned}
i n(|n| \eta+i p) \frac{\partial \tilde{F}_{q}}{\partial j_{n}} & -n \eta j_{-n}-i \sum_{k, l \neq 0} l j_{k} \frac{\partial \tilde{F}_{q}}{\partial j_{-l}} \delta_{k+l+n} \\
& -\sum_{k, l \neq 0}|k| l\left(\frac{\partial \tilde{F}_{q}}{\partial j_{-k}} \frac{\partial \tilde{F}_{q}}{\partial j_{-l}}-i \hbar \frac{\partial^{2} \tilde{F}_{q}}{\partial j_{-k} \partial j_{-l}}\right) \delta_{k+l+n}=0 .
\end{aligned}
$$

\footnotetext{
${ }^{7} F_{q}$ starts at $\mathcal{O}\left(\alpha^{2}\right)$. If one includes both chiral sectors, this becomes $\mathcal{S}=R(p) e^{\frac{i}{\hbar}\left(F_{q}+\bar{F}_{q}\right)}$; see also the discussion in the conclusions.
} 
As before, we make a series Ansatz for $\tilde{F}$, insert it into (5.17) and solve for the coefficients. However, in contrast to the semiclassical case, i.e. as a consequence of the second derivative term in the quantum equation, we can no longer solve for the $\tilde{f}_{n_{1} \ldots n_{\nu}}^{q}$ for generic mode numbers $n_{i}$. But we can still solve them recursively and it is clear that they are rational functions of $p$ and $\hbar$.

For small $\nu$ and small mode numbers one can easily find explicit expressions, which are valid to all orders in $\hbar$. A few examples are ${ }^{8}$

$$
\begin{aligned}
i \tilde{f}_{-11}^{(2, q)} & =\frac{1+\hbar}{1+\hbar+i p}, \quad i \tilde{f}_{-22}^{(2, q)}=\frac{(1+\hbar)\left(2(1+i p)^{2}+(5+4 i p) \hbar+2 \hbar^{2}\right)}{2(1+\hbar+i p)(2+\hbar+i p)(1+2 \hbar+i p)}, \\
i \tilde{f}_{-211}^{(3, q)} & =-\frac{i(1+\hbar) p}{(1+\hbar+i p)(2+\hbar+i p)(1+2 \hbar+i p)}, \\
i \tilde{f}_{-312}^{(3, q)} & =-\frac{i(1+\hbar) p\left((1+i p)^{2}+2(2+i p) \hbar+\hbar^{2}\right)}{(1+\hbar+i p)(2+\hbar+i p)(3+\hbar+i p)(1+2 \hbar+i p)(1+3 \hbar+i p)}, \\
i \tilde{f}_{-1-111}^{(4, q)} & =\frac{2 i(1+\hbar) p}{(1+\hbar+i p)^{2}(2+\hbar+i p)(1+2 \hbar+i p)}, \\
i \tilde{f}_{-2-112}^{(4, q)} & =\frac{2 i(1+\hbar) p(1+i p)(\hbar+i p)}{(1+\hbar+i p)^{2}(2+\hbar+i p)(3+\hbar+i p)(1+2 \hbar+i p)(1+3 \hbar+i p)}, \\
i \tilde{f}_{-3111}^{(4, q)} & =-\frac{2 i(1+\hbar) p(1+\hbar-i p)}{(1+\hbar+i p)(2+\hbar+i p)(3+\hbar+i p)(1+2 \hbar+i p)(1+3 \hbar+i p)} .
\end{aligned}
$$

The expressions with higher mode numbers quickly become too long to display. We have also restricted to at most four oscillator excitations because they are needed for the comparison with the results obtained from the loop calculations. Indeed, expanding to first order in $\hbar$, we find agreement with (5.3) and (5.9). We also found agreement between the $\mathcal{O}\left(\hbar^{2}\right)$ terms in $\tilde{f}_{-11}^{(2, q)}$ and $\tilde{f}_{-22}^{(2, q)}$ and the numerical results of the two-loop amplitudes.

\section{Conclusions}

Our aim in this paper has been the computation of the $S$-matrix of Liouville theory on a cylinder. The analysis of the relation between the asymptotic fields shows that this operator can be represented in the form

$$
\hat{\mathcal{S}}=\hat{\mathcal{P}} R(p) \mathcal{S}_{p}\left(\hat{a}_{n}\right) \mathcal{S}_{p}\left(\hat{\bar{a}}_{n}\right),
$$

where $\hat{\mathcal{P}}$ is the parity operator that reflects the zero modes of the $i n$-field

$$
\hat{\mathcal{P}} \hat{q}_{\text {in }} \hat{\mathcal{P}}=-\hat{q}_{\text {in }}, \quad \hat{\mathcal{P}} \hat{p}_{\text {in }} \hat{\mathcal{P}}=-\hat{p}_{\text {in }},
$$

$R(p)$ is the reflection amplitude $(5.14), \mathcal{S}_{p}\left(\hat{a}_{n}\right)$ depends only on the creation-annihilation operators of the chiral in-field, $\mathcal{S}_{p}\left(\hat{\bar{a}}_{n}\right)$ is its antichiral counterpart and the matrix elements of $\mathcal{S}_{p}\left(\hat{a}_{n}\right)$ in the basis of the coherent states (4.6) are defined by the function of holomorphic variables $\mathcal{S}\left(p, b^{*}, a\right)$ introduced in (4.9). Since the reflection amplitude of Liouville theory is known, the computation of the $S$-matrix is reduced to the analysis of the chiral sector only.

\footnotetext{
${ }^{8}$ The results at levels one and two agree with [6].
} 
The relevance of the computation of the chiral part of the $S$-matrix, given by $\mathcal{S}_{p}\left(\hat{a}_{n}\right)$, goes beyond Liouville theory on the cylinder [14-16] and extends to the theory on the strip [17], which has besides scattering solutions, also bound states [18, 19]. In particular, the asymptotic $i n$-field of Liouville theory on the strip with Neumann boundary conditions has only one set of Fourier modes $a_{n}$ and the $S$-matrix is represented by [20]

$$
\hat{\mathcal{S}}=\hat{\mathcal{P}} R_{b}(p) \mathcal{S}_{p}\left(\hat{a}_{n}\right),
$$

where $\hat{\mathcal{P}}$ is again the parity operator $(6.2), R_{b}(p)$ is the reflection amplitude of the boundary theory and $S_{p}\left(\hat{a}_{n}\right)$ is the same operator as in (6.1).

In contrast to the cylinder, the reflection amplitude on the strip, $R_{b}(p)$ vanishes for a discrete set of purely imaginary momenta, $p=i \theta$, for $\theta<0$, which correspond to bound states. The analytical continuation of the asymptotic fields in this region is given by complex 'free fields', whose real and imaginary parts are related to each other. It was argued in [20] that the analytical continuation of the $S$-matrix to the bound states defines the scalar product between the Fock space vectors of this sector.

To compute the chiral part of the $S$-matrix we have commented on the canonical structure and used it to determine the action $S(\varphi)$ of a one-dimensional theory, which has a non-local first-order kinetic energy term and an exponential potential. It reproduces the scattering amplitudes in the chiral sector, both semiclassical and quantum, via a loop expansion in Feynman diagrams, where the theory is regularised simply by 'normal ordering' the potential. More explicitly, we propose the equation

$$
\int D \varphi e^{-\frac{1}{\hbar}(S(\varphi)-\langle\varphi j\rangle)}=e^{\frac{i}{\hbar} \tilde{F}_{q}(j)}
$$

with $\tilde{F}_{q}$ defined in (5.16) and the sources $j$ are related to the modes of the Liouville scattering problem by a simple rescaling as in (4.22). We proved this at the semi-classical, i.e. tree level and performed some non-trivial checks at the loop level. We consider the identification of the action $S$ as the main result of this paper. It would be interesting to study it further with the aim of computing the complete quantum generating functional.

\section{Acknowledgments}

We acknowledge helpful discussions with Enrico Brehm, Anamaria Font, Antal Jevicki, Axel Kleinschmidt and Evgeny Skvortsov and thank Harald Dorn for discussions and comments on the manuscript. A part of G. J.'s work was done during his visit at Brown University, which was supported by a Fulbright Fellowship. His work was also supported by the joint grant of Volkswagen Foundation and SRNSF (ref. 93562 \& \#04/48).

\section{A Chiral symplectic forms}

The Liouville field and its canonically conjugated momentum are

$$
\begin{aligned}
\Phi(\tau, \sigma) & =\frac{1}{2} \log A^{\prime}(x)+\frac{1}{2} \log \bar{A}^{\prime}(\bar{x})-\log \left[1+\mu^{2} A(x) \bar{A}(\bar{x})\right], \\
\Pi(\tau, \sigma) & =\frac{1}{2} \frac{A^{\prime \prime}(x)}{A^{\prime}(x)}+\frac{1}{2} \frac{\bar{A}^{\prime \prime}(\bar{x})}{\bar{A}^{\prime}(\bar{x})}-\mu^{2} \frac{A^{\prime}(x) \bar{A}(\bar{x})+A(x) \bar{A}^{\prime}(\bar{x})}{1+\mu^{2} A(x) \bar{A}(\bar{x})},
\end{aligned}
$$

where $A$ and $\bar{A}$ are the screening charges of the $i n$-field (see (2.8)-(2.13)). 
The canonical 2-form $\Omega$ defined by the left hand side of (3.3) can be written as

$$
\Omega=\Omega_{0}+\Omega_{1}+\Omega_{2}+\Omega_{3},
$$

with

$$
\begin{aligned}
\Omega_{0}= & \int_{0}^{2 \pi} \frac{\mathrm{d} \sigma}{2 \pi}\left[\left(\frac{\delta A^{\prime}}{2 A^{\prime}}\right)^{\prime} \wedge \frac{\delta A^{\prime}}{2 A^{\prime}}+\left(\frac{\delta \bar{A}^{\prime}}{2 \bar{A}^{\prime}}\right)^{\prime} \wedge \frac{\delta \bar{A}^{\prime}}{2 \bar{A}^{\prime}}\right]+\left.\frac{1}{8 \pi} \frac{\delta A^{\prime}}{A^{\prime}} \wedge \frac{\delta \bar{A}^{\prime}}{\bar{A}^{\prime}}\right|_{\sigma=0} ^{\sigma=2 \pi}, \\
\Omega_{1}= & \int_{0}^{2 \pi} \frac{\mathrm{d} \sigma}{2 \pi}\left[\frac{\mu^{2}}{2}\left(\frac{\delta A^{\prime}}{A^{\prime}}-\frac{\delta \bar{A}^{\prime}}{\bar{A}^{\prime}}\right) \wedge \delta\left(\frac{A^{\prime} \bar{A}-A \bar{A}^{\prime}}{1+\mu^{2} A \bar{A}}\right)\right] \\
& -\left.\frac{1}{4 \pi}\left(\frac{\delta A^{\prime}}{A^{\prime}}-\frac{\delta \bar{A}^{\prime}}{\bar{A}^{\prime}}\right) \wedge \delta\left(\log \left[1+\mu^{2} A \bar{A}\right]\right)\right|_{\sigma=0} ^{\sigma=2 \pi}, \\
\Omega_{2}= & \int_{0}^{2 \pi} \frac{\mathrm{d} \sigma}{2 \pi}\left[\frac{\mu^{2}}{2}\left(\frac{\delta A^{\prime}}{A^{\prime}}+\frac{\delta \bar{A}^{\prime}}{\bar{A}^{\prime}}\right) \wedge \delta\left(\frac{A^{\prime} \bar{A}+A \bar{A}^{\prime}}{1+\mu^{2} A \bar{A}}\right)\right], \\
\Omega_{3}= & \int_{0}^{2 \pi} \frac{\mathrm{d} \sigma}{2 \pi}\left[\frac{\mu^{4}\left(\bar{A} \delta A^{\prime}+A \delta \bar{A}^{\prime}\right) \wedge \delta(A \bar{A})}{\left(1+\mu^{2} A \bar{A}\right)^{2}}+\mu^{2} \frac{\delta \bar{A} \wedge \delta A^{\prime}-\delta \bar{A}^{\prime} \wedge \delta A}{1+\mu^{2} A \bar{A}}\right] \\
& +\left.\frac{\mu^{2}}{2 \pi} \frac{\delta A \wedge \delta \bar{A}}{1+\mu^{2} A \bar{A}}\right|_{\sigma=0} ^{\sigma=2 \pi} .
\end{aligned}
$$

Here, we use the relations $A^{\prime}(x)=\partial_{\sigma} A(x), \bar{A}^{\prime}(\bar{x})=-\partial_{\sigma} \bar{A}(\bar{x})$ and apply partial integrations in $\Omega_{1}$ and $\Omega_{3}$. This extracts the boundary terms in (A.5) and (A.7).

The integrands of $\Omega_{1}, \Omega_{2}$ and $\Omega_{3}$ cancel each other in (A.3). Using the monodromy of the screening charges $A(x+2 \pi)=e^{2 \pi p} A(x), \bar{A}(\bar{x}+2 \pi)=e^{2 \pi p} \bar{A}(\bar{x})$, one finds that the boundary terms of $\Omega_{1}$ and $\Omega_{3}$ cancel each other as well.

Thus, $\Omega=\Omega_{0}=\omega+\bar{\omega}$, with similar chiral and antichiral parts. After the shift of the integration variable $\sigma=x-\tau$, one finds

$$
\omega=\int_{\tau}^{\tau+2 \pi} \frac{\mathrm{d} x}{2 \pi}\left[\left(\frac{\delta A^{\prime}(x)}{2 A^{\prime}(x)}\right)^{\prime} \wedge \frac{\delta A^{\prime}(x)}{2 A^{\prime}(x)}\right]+\frac{1}{2} \delta p \wedge \frac{\delta A^{\prime}(\tau)}{2 A^{\prime}(\tau)} .
$$

This 2-form is $\tau$-independent and in terms of the $i n$-field (2.12) one obtains (3.2).

\section{B Generating function as action functional}

In section 3 we have introduced the generating functionals $G$ and $\bar{G}$. We now describe a different realisation of the generating functions based on the calculation of the action functional on the solutions of the dynamical equations.

Let us consider a Liouville field $\Phi(\tau, \sigma)$ and introduce two free fields $\Phi_{-}(\tau, \sigma)$ and $\Phi_{+}(\tau, \sigma)$, which are tangent to $\Phi(\tau, \sigma)$ at $\tau=\tau_{-}$and $\tau=\tau_{+}$, respectively. We also introduce the actions $S\left[\tau_{+}, \tau_{-}\right], S_{-}\left[\tau_{-}, \tau_{0}\right]$ and $S_{+}\left[\tau_{0}, \tau_{+}\right]$, calculated for $\Phi, \Phi_{-}$and $\Phi_{+}$ respectively, for the corresponding time intervals, i.e

$$
\begin{aligned}
S\left[\tau_{+}, \tau_{-}\right] & =\frac{1}{2} \int_{\tau_{-}}^{\tau_{+}} \mathrm{d} \tau \int_{0}^{2 \pi} \frac{\mathrm{d} \sigma}{2 \pi}\left[\left(\partial_{\tau} \Phi\right)^{2}-\left(\partial_{\sigma} \Phi\right)^{2}-4 \mu^{2} e^{2 \Phi}\right], \\
S_{-}\left[\tau_{-}, \tau_{0}\right] & =\frac{1}{2} \int_{\tau_{0}}^{\tau_{-}} \mathrm{d} \tau \int_{0}^{2 \pi} \frac{\mathrm{d} \sigma}{2 \pi}\left[\left(\partial_{\tau} \Phi_{-}\right)^{2}-\left(\partial_{\sigma} \Phi_{-}\right)^{2}\right], \\
S_{+}\left[\tau_{0}, \tau_{+}\right] & =\frac{1}{2} \int_{\tau_{+}}^{\tau_{0}} \mathrm{~d} \tau \int_{0}^{2 \pi} \frac{\mathrm{d} \sigma}{2 \pi}\left[\left(\partial_{\tau} \Phi_{+}\right)^{2}-\left(\partial_{\sigma} \Phi_{+}\right)^{2}\right] .
\end{aligned}
$$


Using the equations of motion for $\Phi$ and $\Phi_{ \pm}$, together with the identity

$$
\left(\partial_{\tau} \Phi\right)^{2}-\left(\partial_{\sigma} \Phi\right)^{2}=\partial_{\tau}\left(\Phi \partial_{\tau} \Phi\right)-\partial_{\sigma}\left(\Phi \partial_{\sigma} \Phi\right)-\Phi\left(\partial_{\tau}^{2}-\partial_{\sigma}^{2}\right) \Phi
$$

one obtains

$$
\begin{aligned}
S\left[\tau_{+}, \tau_{-}\right] & =I+\frac{1}{2} \int_{0}^{2 \pi} \frac{\mathrm{d} \sigma}{2 \pi}\left[\Phi\left(\tau_{+}, \sigma\right) \partial_{\tau} \Phi\left(\tau_{+}, \sigma\right)-\Phi\left(\tau_{-}, \sigma\right) \partial_{\tau} \Phi\left(\tau_{-}, \sigma\right)\right] \\
S_{-}\left[\tau_{-}, \tau_{0}\right] & =\frac{1}{2} \int_{0}^{2 \pi} \frac{\mathrm{d} \sigma}{2 \pi}\left[\Phi_{-}\left(\tau_{-}, \sigma\right) \partial_{\tau} \Phi_{-}\left(\tau_{-}, \sigma\right)-\Phi_{-}\left(\tau_{0}, \sigma\right) \partial_{\tau} \Phi\left(\tau_{0}, \sigma\right)\right] \\
S_{+}\left[\tau_{0}, \tau_{+}\right] & =\frac{1}{2} \int_{0}^{2 \pi} \frac{\mathrm{d} \sigma}{2 \pi}\left[\Phi_{+}\left(\tau_{0}, \sigma\right) \partial_{\tau} \Phi_{+}\left(\tau_{0}, \sigma\right)-\Phi_{+}\left(\tau_{+}, \sigma\right) \partial_{\tau} \Phi\left(\tau_{+}, \sigma\right)\right]
\end{aligned}
$$

with

$$
I\left(\tau_{+}, \tau_{-}\right)=\int_{\tau_{-}}^{\tau_{+}} \mathrm{d} \tau \int_{0}^{2 \pi} \frac{\mathrm{d} \sigma}{2 \pi}\left[-\frac{1}{2} \Phi\left(\partial_{\tau}^{2}-\partial_{\sigma}^{2}\right) \Phi-2 \mu^{2} e^{2 \Phi}\right] .
$$

Since the fields $\Phi_{ \pm}$are tangent to the Liouville field $\Phi$, from (B.3) we find

$$
\begin{aligned}
& \lim _{t_{ \pm \rightarrow \pm \infty}}\left(S_{0}\left[\tau_{0}, \tau_{+}\right]+S\left[\tau_{+}, \tau_{-}\right]+S_{0}\left[\tau_{-}, \tau_{0}\right]\right) \\
&=2 \mu^{2} \int_{-\infty}^{\infty} \mathrm{d} \tau \int_{0}^{2 \pi} \frac{\mathrm{d} \sigma}{2 \pi} e^{2 \Phi}(\Phi-1) \\
& \quad+\frac{1}{2} \int_{0}^{2 \pi} \frac{\mathrm{d} \sigma}{2 \pi}\left[\Phi_{\text {out }}\left(\tau_{0}, \sigma\right) \partial_{\tau} \Phi_{\text {out }}\left(\tau_{0}, \sigma\right)-\Phi_{\text {in }}\left(\tau_{0}, \sigma\right) \partial_{\tau} \Phi_{\text {in }}\left(\tau_{0}, \sigma\right)\right]
\end{aligned}
$$

The left hand side here corresponds to the generating function (3.14) at $\tau=\tau_{0}$. This leads to

$$
2 \mu^{2} \int_{-\infty}^{\infty} \mathrm{d} \tau \int_{0}^{2 \pi} \frac{\mathrm{d} \sigma}{2 \pi} e^{2 \Phi}(\Phi-1)=G+\bar{G},
$$

where $G$ is the generating function (3.10) and $\bar{G}$ is its antichiral counterpart.

Open Access. This article is distributed under the terms of the Creative Commons Attribution License (CC-BY 4.0), which permits any use, distribution and reproduction in any medium, provided the original author(s) and source are credited.

\section{References}

[1] A.M. Polyakov, Quantum geometry of bosonic strings, Phys. Lett. B 103 (1981) 207 [INSPIRE].

[2] J. Teschner, Liouville theory revisited, Class. Quant. Grav. 18 (2001) R153 [hep-th/0104158] [INSPIRE].

[3] Y. Nakayama, Liouville field theory: A Decade after the revolution, Int. J. Mod. Phys. A 19 (2004) 2771 [hep-th/0402009] [INSPIRE].

[4] N. Seiberg, Notes on quantum Liouville theory and quantum gravity, Prog. Theor. Phys. Suppl. 102 (1990) 319 [INSPIRE].

[5] T. Levy and Y. Oz, Liouville conformal field theories in higher dimensions, JHEP 06 (2018) 119 [arXiv: 1804.02283] [INSPIRE]. 
[6] A.B. Zamolodchikov and A.B. Zamolodchikov, Structure constants and conformal bootstrap in Liouville field theory, Nucl. Phys. B 477 (1996) 577 [hep-th/9506136] [INSPIRE].

[7] J. Balog, L. Feher and L. Palla, Coadjoint orbits of the Virasoro algebra and the global Liouville equation, Int. J. Mod. Phys. A 13 (1998) 315 [hep-th/9703045] [INSPIRE].

[8] J. Liouville, Sur l'équation aux différences partielles $\frac{d^{2} \log \lambda}{d u d v} \pm \frac{\lambda}{2 a^{2}}=0$, J. Math. Pures Appl. 18 (1853) 71.

[9] Y. Nambu, S Matrix in semiclassical approximation, Phys. Lett. B 26 (1968) 626 [INSPIRE].

[10] D.G. Boulware and L.S. Brown, Tree graphs and classical fields, Phys. Rev. 172 (1968) 1628 [INSPIRE].

[11] H. Dorn and H.J. Otto, Two and three point functions in Liouville theory, Nucl. Phys. B 429 (1994) 375 [hep-th/9403141] [INSPIRE].

[12] H. Dorn and H.J. Otto, Remarks on the continuum formulation of noncritical strings, in 26th International Ahrenshoop Symposium on the Theory of Elementary Particles, pp. 0001-11, 12, 1992 [hep-th/9212004] [INSPIRE].

[13] G. Jorjadze and G. Weigt, Poisson structure and Moyal quantization of the Liouville theory, Nucl. Phys. B 619 (2001) 232 [hep-th/0105306] [INSPIRE].

[14] T.L. Curtright and C.B. Thorn, Conformally invariant quantization of the Liouville theory, Phys. Rev. Lett. 48 (1982) 1309 [Erratum ibid. 48 (1982) 1768] [INSPIRE].

[15] E. Braaten, T. Curtright and C.B. Thorn, An exact operator solution of the quantum Liouville field theory, Annals Phys. 147 (1983) 365 [INSPIRE].

[16] H.J. Otto and G. Weigt, Construction of exponential Liouville field operators for closed string models, Z. Phys. C 31 (1986) 219 [INSPIRE].

[17] J.-L. Gervais and A. Neveu, Novel triangle relation and absence of tachyons in Liouville string field theory, Nucl. Phys. B 238 (1984) 125 [INSPIRE].

[18] V. Fateev, A.B. Zamolodchikov and A.B. Zamolodchikov, Boundary Liouville field theory. 1. Boundary state and boundary two point function, hep-th/0001012 [INSPIRE].

[19] B. Ponsot and J. Teschner, Boundary Liouville field theory: boundary three point function, Nucl. Phys. B 622 (2002) 309 [hep-th/0110244] [INSPIRE].

[20] H. Dorn and G. Jorjadze, Operator approach to boundary Liouville theory, Annals Phys. 323 (2008) 2799 [arXiv:0801.3206] [inSPIRE]. 